\title{
Preoperative Evaluation of Venous Systems with Magnetic Resonance Venography in Parasagittal Meningiomas
}

\author{
Esam Elkhatib* and Mohammed Hasanin
}

Department of Neurosurgery, Faculty of Medicine, Suez Canal University, Egypt

\begin{abstract}
Background: Parasagittal meningiomas may pose a difficult surgical challenge because venous patency and collateral anastomoses have to be clearly defined for correct surgical planning. Aim: The aim of this case series was to study the diagnostic accuracy of magnetic resonance venography (MRV) in assessing the superior sagittal sinus (SSS) obstruction and venous collaterals in patients with parasagittal meningioma. Patients and Methods: Seven patients from 2010 through 2012 undergoing surgery for meningiomas located adjacent to the SSS were prospectively evaluated. All patients underwent both conventional magnetic resonance imaging and MRV. Magnetic resonance venography was evaluated to assess: (1) patency of the sinus (Permeable/Occluded), (2) the extent of occlusion (in centimeters), and (3) the number of collateral anastomoses close to the insertion of the meningioma. Magnetic resonance venography assessment was compared with surgery. All patients were operated on, and the intraoperative findings were taken as the reference standard. Results: Magnetic resonance venography showed the sinus to be occluded in 3 cases. MRV data were confirmed by surgery, showing 3 patients to have an occlusion of the SSS. The MRV sensitivity was thus $100 \%$. Magnetic resonance venography recognized $90 \%$ of collateral venous anastomoses close to the meningioma as subsequently confirmed by surgery. Conclusion: In the preoperative planning for patients with meningiomas located close to the SSS, MRV can provide additional and more reliable information concerning venous infiltration, patency of the SSS, and the presence of collateral anastomoses.
\end{abstract}

Keywords: MR venography, venous anastomoses, superior sagittal sinus

\section{Introduction}

Meningiomas account for $15 \%$ to $20 \%$ of all brain tumors, $90 \%$ of which are supratentorial and typically arise along the dural venous sinuses. Parasagittal meningiomas are tumors arising adjacent to the convexity of the skull and are characterized by frequent involvement of the superior sagittal sinus (SSS). In the evaluation of meningiomas involving a venous sinus, the relationship between the lesion nd the adjacent venous structures is crucial for adequate surgical planning ${ }^{(1-4)}$. The importance of preoperative angiographic evaluation for brain tumors has significantly diminished with the availability of magnetic resonance angiography. It is no longer used for the routine diagnosis of brain tumors. In addition, catheter angiography with digital subtraction is invasive and features some complications, including cerebral infarction, injury of vascular walls, and hematoma in the punctured site, which cannot be ignor-

* Corresponding Author: eelkhatib@gmail.com 
ed $^{(5,6)}$. Magnetic Resonance venography (MRV) proved to be a reliable method to distinguish cerebral venous structures. The development of this technology offered a new imaging technique to depict the cerebral vascular circulation. The first applications of MR angiography were for the evaluation of the circle of Willis and the detection of aneurysms. Recently, the venous circulation was also evaluated ${ }^{(7,8)}$. Magnetic resonance venography is currently considered one of the noninvasive tests of choice for evaluation of dural sinuses. MRV affords a rapid method to depict the intracranial venous circulation with consistently high quality ${ }^{(9-12)}$. The purpose of this study was to assess the diagnostic accuracy of MRV in the evaluation of the SSS close to parasagittal meningioma and to evaluate the reliability of MRV in depicting the presence of intracerebral venous structures. Surgery was considered as the reference standard for comparison.

\section{Materials and Methods}

We described seven patients from 2010 through 2012 with parasagittal meningiomas who were candidates for surgical resection; the patients were surgically treated and prospectively evaluated in a period of 9 months at the department of neurosurgery in Suez Canal university hospital. This case series comprised six females $(85 \%)$, and one male (15\%). Five patients (71\%) had middle third lesions, and two patients (29\%) had posterior third lesions. All patients were adults, with a mean age and SD of $57.1 \pm 8.36$ years. The duration of symptoms ranged from 4 months to 21 months, with a mean and SD of $7.3 \pm 5.95$ months. All patients underwent Magnetic Resonance imaging (MRI) examination, including MRV. Image Analysis was done to evaluate each MRV. Three patterns were considered for each venographic tech- nique: 1) Patency of the superior sagittal sinus either permeable or occluded (The SSS was considered occluded when a flow gap inside the sinus was noted). 2) If occluded, the extent of occlusion was estimated in centimeters (assessed as the length of the flow gap inside the sinus). 3) The number of collateral vessels $2 \mathrm{~cm}$ before and $2 \mathrm{~cm}$ after the tumor insertion close to the sinus was counted when this was occluded or clearly infiltrated. Patients underwent surgical intervention for excision of parasagittal meningiomas. During tumor removal, and irrespective to the data obtained from preoperative MRV, sinus patency was evaluated, and when the sinus was occluded, the extent of the occlusion was evaluated through measurement of the length of occlusion or gross tumor invasion. The number of collateral veins bridging the neoplasm or running close to it was also noted.

\section{Results}

Magnetic resonance venography showed 3 occluded sinuses. Surgery confirmed the findings of MRV, demonstrating four permeable and three occluded sinuses. The extent of occlusion was evaluated only in those cases ( 3 patients) confirmed by surgery. The extent of occlusion was evaluated during surgery, and these evaluations led to a total length of the occlusion (sum of all the 3 cases) of $7 \mathrm{~cm}$ (mean $2.3 \mathrm{~cm}$ ). Magnetic resonance venography overestimated the extent of occlusion of the SSS, indicating a total length of $9 \mathrm{~cm}$ (mean $3 \mathrm{~cm}$ ) in the same patients, MRV sequences mildly overestimated occlusion when compared with surgery. A total number of 21 collateral anastomotic veins were seen at surgery (mean 3.5) in the three patients with sinus occlusion and in three patients with sinus. Infiltration (permeable sinus). Assessment of preoperative MRV revealed 
that a total number of 6 collateral anastomotic veins were seen in the two patients with permeable sinus, while a total $13 \mathrm{col}-$ lateral anastomotic veins were seen in the three patients with occluded sinus. Mag- netic resonance venography identified 19 (90\%) of these vessels. The radiographic and intraoperative characteristics of these seven patients are presented in Table 1.

Table 1: Venographic and intraoperative characteristics of parasagittal meningiomas $(\mathrm{N}=7)$

\begin{tabular}{|c|l|l|l|c|c|c|c|}
\hline \multirow{2}{*}{ Patient } & $\begin{array}{c}\text { Location along } \\
\text { SSS }\end{array}$ & \multicolumn{2}{|c|}{ SSS patency } & \multicolumn{2}{c|}{$\begin{array}{c}\text { Extent of } \\
\text { occlusion/cm }\end{array}$} & \multicolumn{2}{c|}{ Collaterals/No } \\
\cline { 3 - 8 } & & MRV & Surgery & MRV & Surgery & MRV & Surgery \\
\hline 1 & Middle & Permeable & Permeable & - & - & 3 & 3 \\
2 & Middle & Occluded & Occluded & 2 & 2 & 4 & 5 \\
3 & Middle & Occluded & Occluded & 4 & 3 & 5 & 5 \\
4 & Middle & Permeable & Permeable & - & - & - & 1 \\
5 & Posterior & Permeable & Permeable & - & - & 3 & 3 \\
6 & posterior & Permeable & Permeable & - & - & - & - \\
7 & Middle & Occluded & Occluded & 3 & 2 & 4 & 4 \\
\hline
\end{tabular}
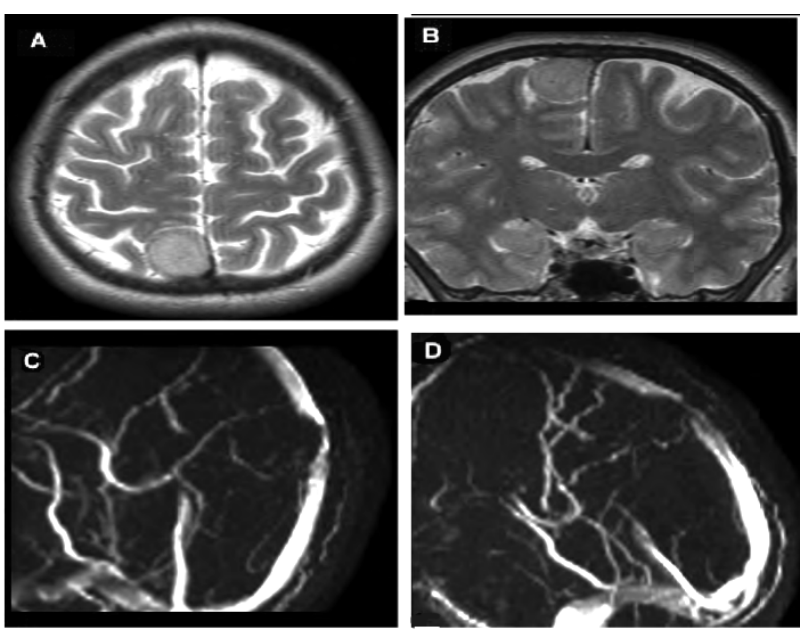

Figure 1: Parasagittal meningioma MRI T2-WI \& MRV. Parasagittal meningioma of the right side. T2-WI $(A, B)$ visualizes the small meningioma adjacent to the wall of the superior sagittal sinus. MR venography(C,D) demonstrates partial obstruction of the sinus lumen in the site of the tumor location.

\section{Discussion}

Parasagittal meningiomas may be a difficult therapeutic challenge, especially when they are located in the middle or posterior third of the SSS. Complete resection of the neoplasm is the treatment of choice, but it might not always be possible if the SSS is infiltrated but still permeable. On the other hand, subtotal resection is frequently asso- ciated with tumor recurrence ${ }^{(4,13,14)}$. When a meningioma is located near the sinus, total resection may require removal of a portion of the SSS, and thus, major concerns include SSS patency, identification of collateral venous pathways, and tumor location. Resection of a patent SSS may be dangerous because surgical obliteration may lead to abrupt venous infarction. Moreover, special attention should be directed to the middle and posterior thirds of the sinus due to presence of important afferent veins draining the precentral and postcentral areas ${ }^{(15)}$. In the case of a completely occluded sinus, progressive occlusion by the tumor allows formation of adequate collateral venous anastomoses, and thus, total removal is advisable. Many reports have emphasized the importance of collateral venous pathways. Subsequently, surgeons should take care in preserving these alternative pathways, which constitute an important collateral circulation. For these reasons, preoperative assessment is fundamental for correct surgical planning, and it should provide the surgeon with all information on location, size, and brain relationship as well as on sinus occlusion and 
collateral veins $^{(8,23)}$. Catheter angiography is still considered the reference standard for the evaluation of arterial and venous vasculature of the brain, but its invasiveness and the procedure-related complications are well known, leading to the common decision of using alternative, noninvasive techniques $^{(16,17)}$.

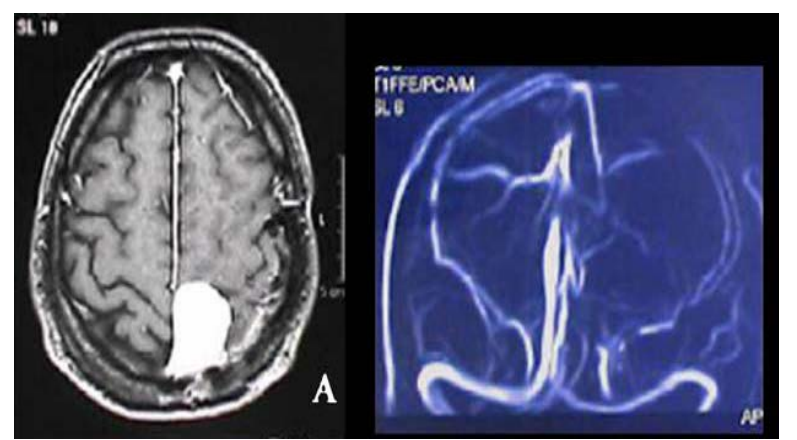

Figure 2: MRI of parasagittal meningioma of middle third of SSS and MRV. A patient with parasagittal meningioma of the middle third of the SSS (A), MRV showing complete occlusion of the sinus (B).

Noninvasive tools for surgical planning of brain tumors are necessary. To obtain necessary information on intracranial venous system, Magnetic resonance Venography (MRV) can be performed as a noninvasive evaluation of intracranial venous anatomy in brain tumors. Previous reports have noted that MRV has a high sensitivity for depicting the intracerebral venous circulation as compared with digital subtraction angiography (DSA) ${ }^{(5)}$. Magnetic resonance venography has already been shown to provide detailed images of the intracranial venous circulation. Contrast-enhanced MRV was shown to be useful in revealing sinus abnormality. In addition, the protocol allows processing of the scan data to create multiplanar reformations in any desired planes as well as $3 \mathrm{D}$ projection images in a virtually limitless number of projections $(18,19)$. In this study, MRV was utilized to assess the venous system of seven selected patients, five of them had tumors located at the middle third, and the other two pa- tients had tumors located at the posterior third of the SSS. Regarding sinus patency, we found that the SSS was permeable in four patients who had middle and posterior third tumors, and totally occluded in three patients all of them had middle third tumors. The extent of occlusion ranged from 2 to $4 \mathrm{~cm}$. Collateral veins in $2 \mathrm{~cm}$ proximity to tumor base on the SSS were counted, number ranged from 3 to 5 collaterals in the total seven cases, but these collaterals seemed more pronounced in case of sinus occlusion than in case of sinus patency.

Bozzao et al in a series of 23 cases suffering parasagittal meningiomas found that contrast-enhanced MRV showed that 5 sinuses were occluded, and 18 sinuses were patent, subsequent surgery confirmed the MRV findings with specificity more than $84 \%$. Bozzao mentioned that up to $87 \%$ of collateral venous anastomoses secondary to parasagittal meningiomas were visualized. Overestimation was mostly observed in the evaluation of the anterior third of the SSS (4/7), being less evident in the middle and posterior third (3/8). The extent of occlusion was evaluated only in those cases (five patients) confirmed by surgery. Length of occlusion measured with mean equals $3.2 \mathrm{~cm}$. A total number of 24 collateral anastomotic veins were seen at surgery (mean 4) in the five patients with sinus occlusion and in three with sinus infiltration without occlusion. Bozzao stated that MRV can provide preoperative insight on venous infiltration and visualize collateral venous anastomoses $^{(15)}$. Jui and colleagues had studied the role of MRV imaging of the intracranial venous system, especially in parasagittal mening-iomas. They concluded that total resection of the tumor may require removal of a portion of the superior sagittal sinus, but in the case of a completely occluded sinus by tumor invasion, sufficient collaterals may have formed to allow total removal of the sinus ${ }^{(20)}$. Despite the valua- 
ble role of MRV in delineating the cerebral venous system, it has some limitations like its affection by the flow-related artifacts, also imaging the uncooperative or moving patient results in motion artifacts. Moreover, MRV is more time consuming and bear difficult monitoring especially in critically ill patients ${ }^{(15)}$. Sindu reported that MRV does not succeed in providing a reliable exploration of the tumor vascularization and hemodynamics, and considered angiography through the trans femoral route is of value prior to establishing the detailed surgical strategy $^{(21)}$. He mentioned that the late venous phases with bilateral filling of the sagittal sinus are required for an exact evaluation of sinus patency and collateral venous pathways $^{(22)}$. In our study, we did not compare MRV with other methods like DSA, the main purpose of our study was to determine the diagnostic accuracy of MRV concerning the SSS patency and collateral veins in parasagittal meningioma patients through subsequent comparison with the intraoperative findings.

\section{Conclusions}

Magnetic resonance venography has proved to be a reliable method to investigate the structure of the SSS and cerebral veins in patients with parasagittal meningiomas, with a reported high sensitivity when compared with surgery as the standard of reference. Owing to its accuracy, non-invasiveness, and ease of interpretation, MRV can be regarded as a valuable preoperative diagnostic method for evaluating the obstruction of the SSS and venous collaterals in patients with parasagittal meningiomas.

\section{References}

1. Buster WP, Rodas RA, Fenstermaker RA, Kattner KA. Major venous sinus resection in the surgical treatment of recurrent ag- gressive dural based tumors. Surg Neurol. 2004; 62 (6):522-530.

2. Akeyson EW, McCutcheon IE. Management of benign and aggressive intracranial meningiomas. Oncology (Williston Park). 1996; 10 (5):747-759.

3. Ayerbe J, Lobato RD, de la Cruz J, Alday R, Rivas JJ, Gómez PA, Cabrera A.. Risk factors predicting recurrence in patients operated on for intracranial meningioma: a multivariate analysis. Acta Neurochir (Wien). 1999;141 (9):921-932.

4. Bonnal J, Brotchi J. Surgery of the superior sagittal sinus in parasagittal meningiomas. J Neurosurg. 1978;48 (6): 935-945.

5. Lee JM, Jung S, Moon KS, Seo JJ, Kim IY, Jung TY, Lee JK, Kang SS. Preoperative evaluation of venous systems with 3dimensional contrast-enhanced magnetic resonance venography in brain tumors: comparison with time-of-flight magnetic resonance venography and digital subtraction angiography. Surg Neurol. 2005; 64 (2):128-134.

6. Haroun A. Utility of contrast-enhanced 3D turbo-flash MR angiography in evaluating the intracranial venous system. Neuroradiology. 2005; 47 (5): 322-327.

7. Wasay M, Azeemuddin M. Neuroimaging of cerebral venous thrombosis. J Neuroimaging. 2005;15 (2):118-128.

8. Leach JL, Fortuna RB, Jones BV, GaskillShipley MF. Imaging of cerebral venous thrombosis: current techniques, spectrum of findings, and diagnostic pitfalls. Radiographics. 2006;26(suppl 1):S19-S41.

9. Widjaja E, Shroff M, Blaser S, Laughlin S, Raybaud C. 2D time-of-flight MR venography in neonates: anatomy and pitfalls. AJNR Am J Neuroradiol. 2006; 27 (9):19131918.

10. Connor SE, Jarosz JM. Magnetic resonance imaging of cerebral venous sinus thrombosis. Clin Radiol. 2002; 57 (6):449-461.

11. Mattle HP, Wentz KU, Edelman RR, Wallner B, Finn JP, Barnes P, Atkinson DJ, Kleefield J, Hoogewoud HM. Cerebral venography with MR. Radiology. 1991; 178 (2):453-458.

12. Kudo K, Terae S, Ishii $A$, , Omatsu $T$, Asano T, Tha KK, Miyasaka K. Physiologic change 
in flow velocity and direction of dural venous sinuses with respiration:MR venography and flow analysis. AJNR Am J Neuroradiol. 2004; 25 (4):551-557.

13. Murata J, Sawamura Y, Saito H, Abe H. Resection of a recurrent parasagittal meningioma with cortical vein anastomosis: technical note. Surg Neurol. 1997; 48(6): 592-597.

14. Palma L, Celli P, Franco C, Cervoni L, Cantore G. Long-term prognosis for atypical and malignant meningiomas: a study of 71 surgical cases. J Neurosurg.1997;86(5): 793-800.

15. Bozzao A, Finocchi V, Romano A, Ferrante $M$, Fasoli F, Trillò G, Ferrante L, Fantozzi $L M$. Role of contrast-enhanced MR venography in the preoperative evaluation of parasagittal meningiomas. Eur Radiol. 2005;15 (9):1790-1796

16. Sajjad Z. MRI and MRV in cerebral venous thrombosis. J Pak Med Assoc. 2006;56(11): 523-526.

17. Nael K, Fenchel M, Salamon N, Duckwiler GR, Laub G, Finn JP, Villablanca JP. Threedimensional cerebral contrast-enhanced magnetic resonance venography at 3.0 Tesla: initial results using highly accelerated parallel acquisition. Invest Radiol. 2006;41 (10):763-768.

18. Rollins N, Ison C, Booth T, Chia J. MR venography in the pediatric patient. AJNR Am J Neuroradiol. 2005;26 (1):50-55.

19. Loubeyre P, De Jaegere T, Tran-Minh VA. Three-dimensional phase contrast MR cerebral venography with zero filling interpolation in the slice encoding direction. Magn Reson Imaging. 1999;17 (8):1227-1233.

20. Fu JH, Lai PH, Hsiao CC, Li SC, Weng MJ, Wang PC, Chen CK. Comparison of realtime three-dimensional Gadolinium-enhanced elliptic centric-ordered MR venography and two-dimensional Time-of-flight MR venography of the Intracranial Venous System. J Chin Med Assoc:2010:73 (3);131138.

21. Alvernia JE, Sindou MP. Preoperative neuroimaging findings as a predictor of the surgical plane of cleavage: prospective study of 100 consecutive cases of intracra- nial meningioma. J Neurosurg. 2004:100 (3);422-430.

22. Sindou $M$. meningiomas involving the major dural sinuses: management of the sinus invasion. in: Practical Handbook of Neurosurgery ; Springer-Verlag 2009; 175-179. 